of the night sky has been indicated recently, and that its intensity would exceed that of the green line of [O I]. This fact, important for the physics of the upper atmosphere, confirms the ideas recently expounded by one of us ${ }^{4}$, who showed that nitrogen must be found essentially in the atomic state in the upper atmosphere. But it remains possible that the two identifications that we suggest may be simultaneously true; moreover, the two emissions may be bound together by a mechanism one of us has already pointed out ${ }^{5}$ : the $(0,0)$ band of the first positive system corresponds to the $B_{0}-A_{0}$ transition of the nitrogen molecule; now the presence of the Vegard-Kaplan bands in the radiation of the night sky leads us to admit the presence of numerous $A_{0}$ molecules in the upper atmosphere; through the collision between the $A_{0}$ molecules and the ${ }^{2} P$ atoms we can have the following process :

\begin{tabular}{|c|c|c|}
\hline 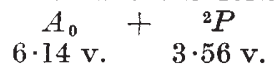 & $\rightarrow \quad \begin{array}{c}B_{0} \\
7 \cdot 32 \mathrm{v} .\end{array}$ & $+\quad \begin{array}{c}2 D \\
2 \cdot 37 \mathrm{v}\end{array}$ \\
\hline $9 \cdot 70 \mathrm{v}$ & & $\begin{array}{l}\cdot 69 \mathrm{v} . \\
\text { R. HERMAN } \\
\text { L. HERMAN. } \\
\text { J. GAUZIT. }\end{array}$ \\
\hline
\end{tabular}

Observatoire de Lyon,

Saint-Genis-Laval,

Rhone.

Feb. 19

"Herman, R., Herman, L., and Gauzit, J., "Cahiers de Physique" No. 12,46 (1942).

'Cabannes, J., J. Phys., 5, 601 (1934).

spetker, A. H., Phys. Rev., 30, 823 (1937).

'Gauzit, J., C.R. Acad. Sci., 213, 695 (1941); "Cahiers de Physique", No. 9,47 (1942)

- Herman, Mme. R., C.R. Acad. Sci., 212, 120 (1941)

\section{Behaviour of Ultra-Violet and Daylight Rays in the Solar Cycle}

WHEN graphs are constructed, for the sunspot cycle 1933-44, of the annual averages both of ultraviolet and of daylight rays, they are found to have a decisive minimum at the sunspot maximum, of 1937-38, and the drop from maximum to minimum of the daylight curves is approximately twice as large as that of the ultra-violet curves. This drop of the rays received at the time when the sun has its highest activity, as shown by the maximum number of spots, suggests that the rays emitted from the sun are in part absorbed in their passage to the earth by some agency which fluctuates with the sun's activity and is most effective at the time of maximum sunspots. The ionization of the upper air due to ultra-violet rays is a probable agency in producing this effect.

While the daylight rays show an inverse relation to the sunspot curve and have a period the same as the sunspots, the ultra-violet rays exhibit two weak maxima on either side of its minimum and the curve appears to have a double period in the solar cycle. An explanation of these observations can be given on the following lines.

As regards the daylight rays, if they are not subject to any large fluctuations on emission from the sun, but are impeded in their passage to the earth by an agency of period like that of the sunspots, such as ionization of the upper air, they will have a single period in sympathy with the solar cycle. If, on the other hand, rays such as ultra-violet rays coming from the sun are subject to sunspot activity and fluctuate with it, and if these rays generate the fluctuating ionization already referred to, which at the same time acts as an absorbing agent, then the ultra-violet rays received on the surface of the earth would at first increase with growing sunspots; but at an early stage in the cycle the impeding ionization would reduce their intensity and a point would be reached when they would begin to diminish and continue to do so as far as their minimum when ionization is greatest; after which a reverse action would ensue, the rays would increase, due to reduction of ionization, and at a later stage they would diminish as their emission from the sun fell off, and two weak maxima would be presented one on each side of the droop of the curve. Thus the single period of the daylight rays coinciding with the period of the solar cycle would be explained by the emission of rays nearly constant over the solar cycle, while the double maximum of the ultra-violet rays would occur if the rays are not emitted uniformly but are subject to a fluctuation in sympathy with the sun's activity.

One result of this point of view is that at an early stage and again at a later stage in the solar cycle the received daylight and ultra-violet rays should move in opposite directions. In the latter part of the solar cycle of 1933-44 this comes out clearly, daylight rays increasing and ultra-violet rays decreasing simultaneously.

At the present time, sunspots are a minimum and there will probably be a rapid increase in their number in the next three or four years. These years will be of the highest interest in verifying or, if necessary, modifying the conclusions derived from the observations made during the solar cycle of 1933-44.

55 King Street South, J. R. Ashworth. Rochdale. May 25.

\section{Inverse Binomial Sampling}

IN a recent communication discussing the application of the sampling method described by Haldan ${ }^{1}$, Tweedie ${ }^{2}$ states that the method was described as a technique for using hæmocytometers. This is not so. Hæmocytometers are designed for estimating the population density per unit volume, and the method under discussion is adapted for estimating the incidence of an attribute in a population consisting of members possessed of that attribute and members not possessed of it. This is an entirely different problem. The method, which has been in use in this Laboratory for a considerable time, and has proved of great value, was designed for estimating the incidence of certain abnormal forms of erythrocytes in blood films, this incidence often being as low as $0 \cdot 2$ per cent. By using this sampling technique, increased accuracy is obtained at these low incidence levels, and a saving of time and labour occurs at high incidence levels.

As both Haldane and Tweedie indicate, the method has much wider applications than to hæmatological estimations, but it does not extend to the hæmocytometer type of sampling.

\section{R.N. Physiological Laboratory, Clayhall Road, \\ Alverstoke, Hants.} R. A. M. CASE. April 27.

1 Haldane, J. B. S., Nature, 155, 49 (1945). 'Tweedie, M. C. K., Nature, 155, 453 (1945). 